\title{
HPVI6E7d candidate vaccine modified genes expression in tumor environment: flic as adjuvant changed tgf-b gene expression
}

\begin{abstract}
Cervical cancer is one of the most important venereal disease and leads to mortality in women. Human papilloma virus causes this disease. This virus has three protein zones that includes of Early, Late and None coding areas. The early phase consists of E1 to E7 but E6 and E7 are two early oncoproteins of HPV which are responsible for disordering in cell cycle and tumor induction. In this study, the efficiency of FlicE7d-HPV16 and HPV16-E7d-Flic were investigated in tumor bearing Mice models .Tumor was induced in mice with transplantation method. After tumor growth, the mice were divided into five groups. two groups of C57BL/6 Mice were injected with $20 \mu \mathrm{g}$ of the vaccine Flic-E7d-HPV16 and E7d-HPV16-Flic subcutaneously three times with one week interval. The third group only E7d was injected and for fourth group only Flic was injected and for fifth group PBS was injected. One week after final immunization, tumor was assessed for gene expression and pathologic smear. According to pathological studies on the tumor samples of mice which indicated the tumor was kind of Sarcoma, Necrosis, Apoptosis, Mitosis and vessel generation were evaluated in each group. Apoptosis and angiogenesis in A, B group were not observed. According to investigation of TGF- $\beta$ and VEGF genes and HPRT control gene using PCR-real-Time, there was a decrease of VEGF and TGF- $\beta$ genes in group A and B. SO the vaccine of group A, B were potent in the reduction of angiogenesis. As a result, Flic as an adjuvant improved anti-tumor effect of HPV16 E7d vaccine.
\end{abstract}

Volume 2 Issue 5 - 2017

\author{
Behazin Aria,' Nasser Rakhshani, ${ }^{2}$ Farhad \\ Riazirad, ${ }^{3}$ Mehdi Mahdavi, ${ }^{3}$ \\ 'Department of Microbiology, Islamic Azad University, Iran \\ ${ }^{2} \mathrm{Gl}$ and Liver Disease Research Center, Iran University of \\ Medical Sciences, Iran \\ ${ }^{3}$ Department of Immunology, Pasteur Institute of Iran, Iran
}

Correspondence: Mehdi Mahdavi, Departments of Immunology, Pasteur Institute of Iran, Tehran, Iran, 69 Pasteur Ave Kargar Ave, PO Box 131694355 I, Tel/Fax +98 2I 64I I2840, Email M Mahdavi@pasteur.ac.ir; Mahdavivac@gmail.com

Received: October 31, 2017 | Published: November 24, 2017

Keywords: HPV16E7d flic, gene expression, pathology

\section{Introduction}

Cervical cancer is one of the most important disease leads to mortality in women the entire world. HPV is directly related to cervical cancer. ${ }^{1}$ This virus has three protein regions that include early, late and non-coding areas. The early region consist of E1 to E7 that E6 and E7 are two main oncoproteins of HPV, which are responsible for disturbance in cell cycle and tumor progression of infection. ${ }^{1}$ E7 protein with binding to retinoblastoma protein result in uncontrolled cell mitosis..$^{2-5}$ So that, Because of the risk of oncogenic, it was utilized as a type of mutant protein with three substitution in amino acids. Therefore called E7 detox (E7d). While changes have been prevented from binding proteins to Retinoblastoma protein $(\mathrm{pRb})$, it has been maintained in immunogenic property. Carcinogenesis potency of high risk Papilloma virus is associated with E6 and E7 protein that is expressed in cancerous cells constantly. Although these proteins are small, they have high capacity in binding to regulatory proteins of host cell to create malignancy in target tissues..$^{6-9}$ Todays, it is believed that a bare protein is not enough immunogenic and should be formulated in adjuvants. Actually adjuvants are some substances that increase vaccine immunogenicity and thereby vaccine potency. Adjuvants used in combination or mixture with vaccines by various mechanisms improve immune responses. ${ }^{10,11}$ Such adjuvants are microbial product which bind to the Toll like receptors and induce inflammation. The microbial products that bind to the TLR5 is flagellin (FliC). In fact, Flic as adjuvant is able to bind to TLR5 on dendritic cells and macrophages. ${ }^{12,13}$ So that, some signals are generated to activate adaptive immune system. ${ }^{13,14}$ Some studies showed that
VEGF could induce angiogenesis through effect on endothelial cells directly and also facilitate access oxygen and nutrients to tumor growth. VEGF plays a central role as a mediator in tumor growth and angiogenesis. ${ }^{15,16}$ Another factor that should be considered as tumor progression is TGF- $\beta$. TGF- $\beta$ suppresses immune responses in favor of tumor growth. On the other hand, studies show that tumor cells with secretion of TGF- $\beta$ could escape from immuno surveillance mechanisms. ${ }^{17}$ Until now, different procedures were utilized to create therapeutic vaccines against of human Papilloma virus such as DNA, peptide, protein, whole cell vaccines. Todays, utilization of TLRs agonists is a method to increase vaccine efficacy. ${ }^{13,14}$ Herein, type B pseudomonas aeruginosa recombinant $\mathrm{FliC}$ was used as adjuvant. In this study, Flic-E7d-HPV16, HPV16-E7d-Flic protein efficacy was investigated as vaccines in tumor bearing mice models. Also, tumor environment events were investigated, furthermore, after vaccination, TGF- $\beta$, VEGF genes expression level and apoptosis, necrosis, mitosis and angiogenesis was appraised in pathologic slides.

\section{Material and methods}

\section{Mice}

Female six-to-eight week old inbred C57BL/6 mice were purchased from Pasteur Institute of Iran (Karaj) and kept them for one week before experiment. Mice were kept in animal room with suitable ventilation at $20-22^{\circ} \mathrm{C}$. Given free access to water and food and maintained in a $12 \mathrm{hr}$ light $/ 12 \mathrm{hr}$ dark cycles. Handling of the mice was carried out with a professional technician and in accompanied by the Animal Care and Use Protocol of Pasteur Institute of Iran. 


\section{Tumor modeling}

Tumor was induced by cell line injection manner using TC1 cell line. Briefly, after cell suspension preparation of TC1 cell line, the cells washed and PBS was added and suspension adjusted to $1 \times 10^{7}$ cells $/ \mathrm{ml}$. finally, one million cells was injected at the flank of C57BL mice. After two weeks. Tumor developed and used of as stock for induction of tumor induction in native mice via transplantation method. Briefly tumor dissected to 3-5 $\mathrm{mm}$ pieces and transplanted to the flask of anesthetized mice.

\section{Vaccine preparation}

Flic-E7d, E7d-flic, Flic and E7d recombination protein was produced and purified by Meysam Gachpazan at Department of Virology from Pasteur Institute of Iran and gifted for this study. Flic protein was utilized as a recombination flagellin of pseudomonas aeruginosa which was produced previously by Dr. Sobhan Faezi ${ }^{18}$ from Pasteur Institute of Iran.

\section{Study groups and immunization}

Tumor Bearing mice were divided into five groups. Two groups of tumoral C57BL/6 mice were injected with $20 \mu \mathrm{g}$ of vaccine FlicE7d-HPV16, and E7d-HPV16-Flic subcutaneously, for third group only E7d was injected and fourth group only Flic was injected and for fifth group control PBS was injected. Adjuvant (Flic) and PBS as control groups. Each group was marked by punctuation on the ear. Immunization was done three times with one week interval. After last inoculation, tumor samples of experimental mice removed and analyzed for gene expression and pathology parameters.

\section{Primer design}

Primers (VEGF1 forward 5'-TGT ACC TCC ACC ATG CCA AGT-3'; VEGF1 reverse 5'-TGG TAG ACG TCC ATG AAC TTG3'), (VEGF2 forward 5'-GGA GAT CCT TCG AGG AGC ACT-3'; VEGF2 reverse 5'-GGC GAT TTA GCA GCA GAT ATA AGA-3') and (TGF $\beta$ forward 5'-CAC CTG CAA GAC CAT CGA CAT-3'; TGF $\beta$ reverse $5^{\prime}$-GAG CCT TAG TTT GGA CAG GAT CTG-3') were designed to amplify $145,94,130$ and 85 base pairs fragments respectively. These small primers (less than $250 \mathrm{bp}$ ) were attached to mRNA on the border between two exons. All of the primers were appraised with primer express 3.0 software and adopted among papers. Selected primers sequences were tested by blast software.

\section{RT- PCR assay}

The method was performed according to the optimum set up of our laboratory. Briefly, RNA of tumor samples was extracted with RNX Plus (Qiagen) and cDNA was synthesized. Gene expression was assessed by Real Q-PCR 2x Master Mix Kit. Along with, SYBR green was used to identify amplified PCR production. Also, $5 \mu \mathrm{L}$ of SYBR Taq II, $1 \mu \mathrm{L}$ of PCR forward primer, $1 \mu 1$ of PCR reverse primer, $1 \mu \mathrm{l}$ of Template, $2 \mu \mathrm{l}$ of $\mathrm{dH} 2 \mathrm{O}$ in reaction mixture was used. To carry out Real time PCR reaction, Rotor Gene 6000 thermal cycler device (Corbett Company) was used. Temperature plane includes the following steps: initial denaturation $\left(95^{\circ} \mathrm{C}, 15 \mathrm{~min}\right)$ denaturation $\left(95^{\circ} \mathrm{C}, 10 \mathrm{~s}\right)$, annealing and extension $\left(62^{\circ} \mathrm{C}, 20 \mathrm{~s}\right)$ and the steps consist of 40 cycles. In other to properties evaluation of PCR, Melting curve was used at $43^{\circ} \mathrm{C}$. RT-PCR method was optimized with primer binding temperature and primer concentration optimization.

\section{Smear pathology preparation}

Experimental tumor samples were placed in Tissue-Process for 12h. After that, the fixed samples are ready to section and were stained by H\&E. eventually; prepared slides were investigated at Pathology Department of Firoozgar Hospital (Tehran, Iran).

\section{Statistical analysis}

All analysis was carried out by Graph pad prism V6.01 software and ANOVA test for statistical analysis and $\mathrm{P}$ value $<0.05$ was considered as significant difference.

\section{Results}

\section{Real time PCR}

Results of VEGF gene expression showed significant decrease in Flic-E7d, E7d-Flic and E7d vaccine candidate groups as in comparison to PBS and Flic control groups $(\mathrm{p}<0.0005)$ (Figure 1). Also, the VEGF gene expression level in the groups immunized with Flic-E7d, E7d-Flic and E7d did not show significant differences $(p>0.05)$. Results of TGF- $\beta$ gene expression showed significant decrease in Flic-E7d vaccine candidate group as compared with PBS control group $(\mathrm{p}=0.004)$. Insignificant decrease was observed in FlicE7d vaccine candidate group in compared to E7d-Flic group ( $\mathrm{p}=0.44)$. TGF- $\beta$ gene expression at group received vaccine formulated with Flic-E7d does not show significant differences versus Flic control group ( $\mathrm{p}>0.05$ ). Evaluation of TGF- $\beta$ gene expression results at group immunized with E7d-Flic versus E7d vaccine candidate group did not show significant difference $(\mathrm{p}>0.05)$. Also, immunization with E7d-Flic showed decrease versus PBS control group, however that difference was not significant ( $\mathrm{p}>0.05$ ). Group received E7d didn't show significant differences as compared with Flic and PBS control groups ( $>0.05$ ) (Figure 2).

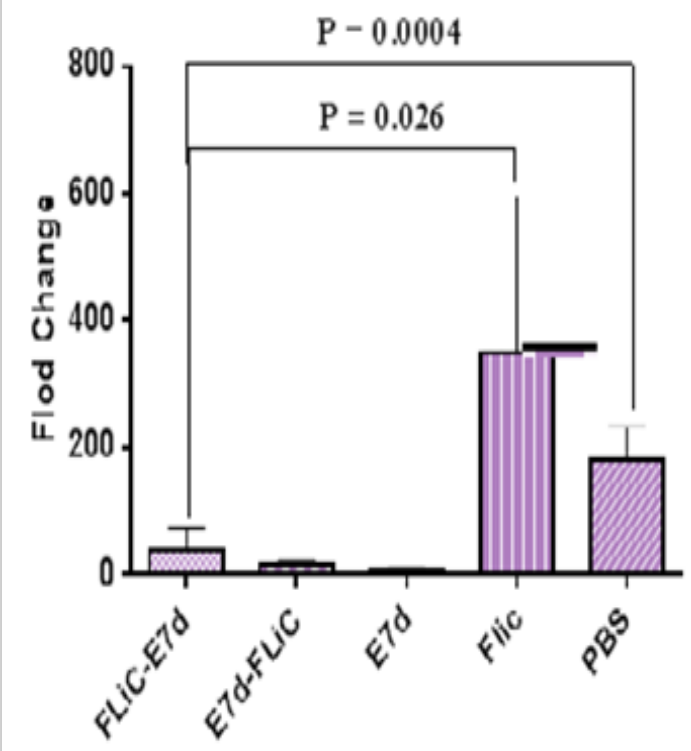

\section{Experimental Groups}

Figure I Result of VEGF gene expression analysis after immunization course. Results ofVEGF gene expression showed significant decrease in Flic-E7d, E7dFlic and E7d vaccine candidate groups as in comparison to PBS and Flic control groups $(p<0.0005)$. 


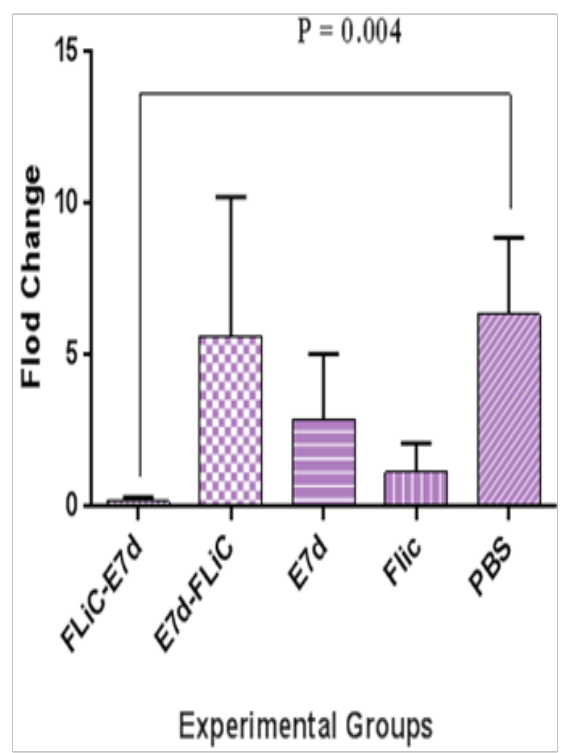

Figure 2 Real time-PCR analysis ofTGF- $\beta$ gene expression in the experimental groups. Results of TGF- $\beta$ gene expression showed significant decrease in FlicE7d vaccine candidate group as compared with PBS control group $(p=0.004)$. Significant decrease was not observed in Flic-E7d vaccine candidate group as compared to E7d-Flic group $(p=0.44)$.

\section{Pathologic results}

Apoptosis: Evaluation of apoptosis Results shows that immunization with Flic-E7d cause significant increase apoptosis versus control groups (Flic vaccine and $\mathrm{PBS}$ ) $(\mathrm{P}=0.0012, \mathrm{P}=0.0185$ respectively). Also, immunization with E7d-Flic vaccine candidate shows significant increase of apoptosis level versus Flic control group ( $\mathrm{P}=0.0186)$. Apoptosis level at group received E7d vaccine has increased as compared with $\mathrm{PBS}$ and Flic control groups $(\mathrm{P}=0.0437, \mathrm{P}=0.003$ respectively). The apoptosis level at Flic-E7d, E7d and E7d-Flic groups shows no significant differences versus each other $(\mathrm{p}>0.05)$ (Figure 3$)$.

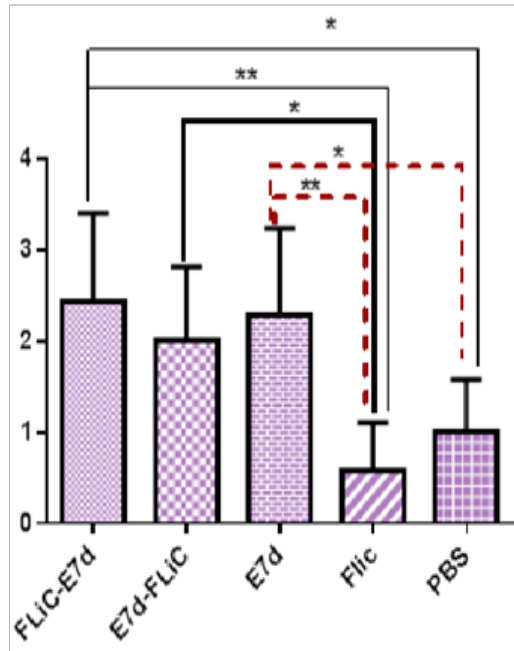

Experimental Groups

Figure 3 Assessment of Apoptosis levels in experimental groups. Immunization with Flic-E7d cause significant increase apoptosis versus Flic and PBS groups $(P=0.00 I 2, P=0.0185$ respectively). Also, immunization with E7d-Flic vaccine candidate show significant increase of apoptosis level versus Flic control group $(P=0.0186)$. The apoptosis levels at Flic-E7d, E7d and E7d-Flic groups show no significant differences versus each other
Necrosis: Necrosis results showed that immunization with E7d-Flic significantly increases necrosis percent versus other experimental groups (Flic-E7d, Flic, E7d, PBS) $(\mathrm{P}<0.05)$. Necrosis percent at group received Flic-E7d didn't show significant differences as compared with Flic, E7d and PBS groups ( $p>0.05$ ). Immunization with Flic-E7d does not show significant differences when compared with E7d, Flic and PBS control groups ( $\mathrm{P}>0.05)$. (Figure 4).

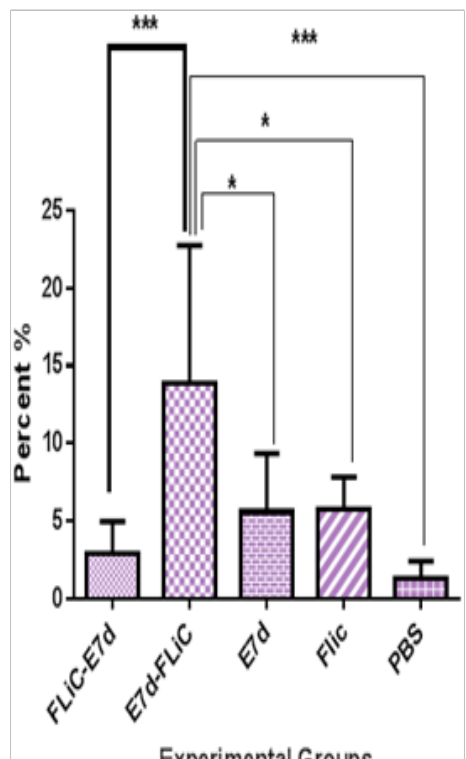

Experimental Groups

Figure 4 Analysis of necrosis levels in experimental groups. Immunization with E7d-Flic significantly increase necrosis percent versus other experimental groups (Flic-E7d, Flic, E7d, PBS) $(P<0.05)$.

Mitosis: Immunization with Flic-E7d significantly decreases mitosis percent versus E7d-Flic and E7d groups $(\mathrm{P}<0.0004)$ but does not shows significant differences versus PBS and FliC control groups $(\mathrm{P}>0.05)$. Immunization with E7d-Flic shows significant increase versus PBS and FliC control groups $(\mathrm{P}<0.0001)$, but not versus E7d group ( $\mathrm{P}>0.05)$ (Figure 5).

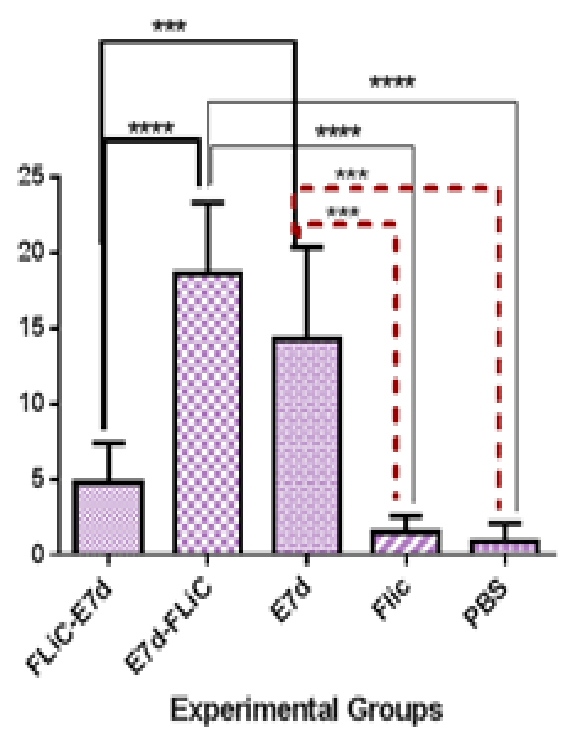

Figure 5 Assessment of mitosis levels in experimental groups. Immunization with Flic-E7d significantly decrease mitosis percent versus E7d-Flic and E7d groups $(\mathrm{P}<0.0004)$. 
Angiogenesis: Immunization with Flic-E7d and E7d-Flic show a significant decrease angiogenesis percentage versus E7d vaccine group $(\mathrm{P}<0.0042)$. But, immunization with Flic-E7d and E7d-Flic does not show significant differences as compared with Flic and PBS control groups $(\mathrm{P}>0.05)$. Immunization with $\mathrm{E} 7 \mathrm{~d}$ significantly increase angiogenesis percentage versus Flic and PBS control groups $(\mathrm{P}=0.0042)$ (Figure 6).

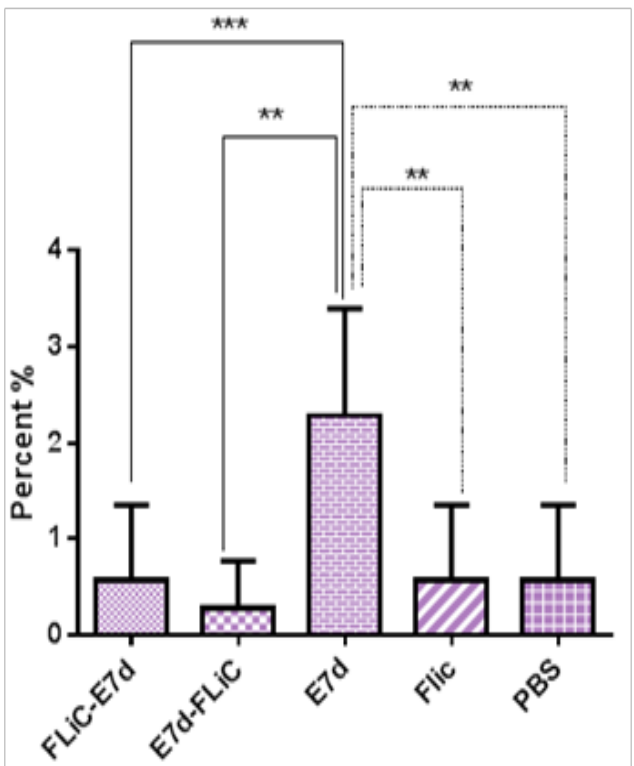

Experimental Groups

Figure 6 Results of angiogenesis percent in experimental groups. Immunization with Flic-E7d and E7d-Flic show a significant decrease angiogenesis percentage versus E7d vaccine group $(P<0.0042)$.

\section{Discussion}

In the present study, HPV vaccine with E7d mutated protein of type 16 papilloma virus accompanied with Flic adjuvant was used as a model to descript of immunization versus HPV vaccine candidate in tumor bearing mice. E7d protein is expressed in tumor cells constantly and is considered to cancer treatment followed by HPVs infection. ${ }^{7}$ Herein, we evaluated TGF-B cytokine gene expression as key immuno-suppressor cytokine in tumor milieu and also apprised pathology markers that reflect immune responses against tumor tissue. Our results show that administration with Flic-E7d vaccine candidate significantly decreased level of VEGF and TGF- $\beta$ genes expression. Several studies show that VEGF is up-regulated by hypoxia and some inflammatory cytokines and also through effects on endothelial cells could induce angiogenesis and promote tumor growth. Along with, studies show that TGF- $\beta$ as a potent regulator factor is considered to suppress immune system and important promoter of cancerous cell growth and progression. ${ }^{19}$ Elevated TGF- $\beta$ converted naïve T cells to regulatory $\mathrm{T}$ cells that in turn provide immune response escape and tumor progression..$^{20}$ Also, Branca et al. ${ }^{21}$ showed that VEGF increased in cervical cancer, and related to high risk HPV in cervical lesions closely. ${ }^{21}$ Boil et al. ${ }^{20}$ showed that VEGF was elevated in patient with cervical cancer. Yang et al. showed that TGF- $\beta$ promote tumor growth through changing microenvironment and establishment of suppress condition in immune system [20]. So that, VEGF and TGF- $\beta$ which are really vital to tumor survival and growth are compatible with other study in this respect. Therefore, our results showed that Flic-
E7d could inhibit immune response suppression and tumor growth by decrease of VEGF and TGF- $\beta$ even than E7d vaccine that in turn reflects positive effect of FliC as adjuvant that modified immune response in the tumor microenvironment. Results of pathology experiments showed that Flic-E7d, E7d-Flic, and E7d candidate vaccine groups significantly increased level of apoptosis as compared with control groups. Moreover, E7d-Flic could significantly decreased angiogenesis versus E7d candidate vaccine group and also Flic-E7d significantly decreased anaplasia percent as compared with two other vaccinated groups. Along with, E7d-Flic significantly increased necrosis percent versus all experimental groups. The apoptosis and necrosis mechanism implicated in tumor elimination ${ }^{22}$ as here FliC as adjuvant in E7d vaccine increased these parameters.

Also, studies showed that blood vessels formed by adequate nutrients and oxygen are called angiogenesis by which leads to metastasis of tumor cells. In addition, some studies demonstrated that, in cancer condition mitosis was forced to P53 arrest and followed by this situation uncontrolled proliferation occurred. ${ }^{23,24}$ Herein, E7dFlic and Flic-E7d could decrease significantly angiogenesis percent and efficacy of angiogenesis suppression in these candidate vaccine groups was higher than E7d vaccine. Inhibition of tumor angiogenesis is an important mechanism in tumor treatment via immunotoxins. ${ }^{25}$ From this regard, we have shown that E7d as vaccine candidate and Flic as a benefit adjuvant be able to increase of apoptosis, necrosis, and decrease in mitosis and angiogenesis in the tumor tissue samples, also FliC as adjuvant could affects on VEGF and TGF-B genes expression in tumor environment and suppress angiogenic and immunosuppressive TGF-B cytokine in tumor milieu. According to what was mentioned, E7d-Flic and Flic-E7d as well could limit angiogenic and TGF-B cytokine gene expression in tumor tissue samples. Taken together, these results indicate that the present vaccine could be a candidate to use as therapeutic vaccine.

\section{Acknowledgment}

We thank Dr. Hossein Keymosy, Mr. Mehdi Adib for technical support. This work was supported in part by a grant from Pasteur Institute of Iran, Tehran, Iran.

\section{Conflict of interest}

Authors declare no conflict for this work.

\section{References}

1. Poljak M. Review of 20 years of HPV research in slovenia. Acta Dermatovenerol Alp Pannonica Adriat. 2011;20(3):99-112.

2. Burk RD, Harari A, Chen Z. Human papillomavirus genome variants. Virology. 2013;445(1-2):232-243.

3. Doorbar J, Quint W, Banks L, et al. The biology and life-cycle of human papillomaviruses. Vaccine. 2012;30(Suppl 5):F55-70.

4. Liu X, Clements A, Zhao K, et al. Structure of the human Papillomavirus $\mathrm{E} 7$ oncoprotein and its mechanism for inactivation of the retinoblastoma tumor suppressor. J Biol Chem. 2006;281(1):578-586.

5. Zur Hausen H. Papillomaviruses and cancer: from basic studies to clinical application. Nat Rev Cancer. 2002;2(5):342-350.

6. Bachmann M, Horn K, Poleganov MA, et al. Interleukin-18 secretion and Th1-like cytokine responses in human peripheral blood mononuclear cells under the influence of the toll-like receptor-5 ligand flagellin. Cell Microbiol. 2006;8(2):289-300. 
7. Barbosa MS, Lowy DR, Schiller JT. Papillomavirus polypeptides E6 and E7 are zinc-binding proteins. J Virol. 1989;63(3):1404-1407.

8. Carter JR, Ding Z, Rose BR. HPV infection and cervical disease: a review. Aust N Z J Obstet Gynaecol. 2011;51(2):103-108.

9. El Bakkouri K, Descamps F, De Filette M, et al. Universal vaccine based on ectodomain of matrix protein 2 of influenza A: Fc receptors and alveolar macrophages mediate protection. J Immunol. 2011;186(2):10221031.

10. Jamali A, Mahdavi M, Hassan ZM, et al. A novel adjuvant, the general opioid antagonist naloxone, elicits a robust cellular immune response for a DNA vaccine. Int Immunol. 2009;21(3):217-225.

11. Jazani NH, Parsania S, Sohrabpour M, et al. Naloxone and alum synergistically augment adjuvant activities of each other in a mouse vaccine model of Salmonella typhimurium infection. Immunobiology. 2011;216(6):744-751.

12. Gewirtz AT, Navas TA, Lyons S, et al Cutting edge: bacterial flagellin activates basolaterally expressed TLR5 to induce epithelial proinflammatory gene expression. J Immunol. 2001;167(4):1882-1885.

13. Ivison SM, Khan MA, Graham NR, et al. A phosphorylation site in the Toll-like receptor 5 TIR domain is required for inflammatory signalling in response to flagellin. Biochem Biophys Res Commun. 2007;352(4):936-941.

14. Tino T. Genetics of structure and function of bacterial flagella. Annual review of genetics. 1977;11:161-182.

15. Carmeliet P. VEGF as a key mediator of angiogenesis in cancer. Oncology. 2005;69(Suppl 3):4-10.

16. Harris AL. Hypoxia-a key regulatory factor in tumour growth. Nat Rev Cancer. 2002;2(1):38-47.
17. De Visser KE, Kast WM. Effects of TGF- $\beta$ on the immune system: implications for cancer immunotherapy. Leukemia. 1999;13(8):1188-99.

18. Faezi S, Safarloo M, Amirmozafari N, et al. Protective efficacy of Pseudomonas aeruginosa type-A flagellin in the murine burn wound model of infection. APMIS. 2013;122(2):115-127.

19. De Visser KE, Kast WM. Effects of TGF- $ß$ on the immune system: implications for cancer immunotherapy. Leukemia. 1999;13(8):1188-1199.

20. Yang P, Li QJ, Feng Y, et al. TGF- $\beta$-miR-34a-CCL22 signaling-induced Treg cell recruitment promotes venous metastases of HBV-positive hepatocellular carcinoma. Cancer cell. 2012;22(3):291-303.

21. Branca M, Giorgi C, Santini D, et al. Aberrant expression of VEGF-C is related to grade of cervical intraepithelial neoplasia (CIN) and high risk HPV, but does not predict virus clearance after treatment of CIN or prognosis of cervical cancer. J Clin Pathol. 2006;59(1):40-47.

22. Lowe SW, Lin AW. Apoptosis in cancer. Carcinogenesis. 2000;21(3):485-495.

23. Kuffer C, Kuznetsova AY, Storchová Z. Abnormal mitosis triggers p53-dependent cell cycle arrest in human tetraploid cells. Chromosoma. 2013;122(4):305-318.

24. Prager G, Poettler M. Angiogenesis in cancer. Hämostaseologie. 2012;32(2):105-114.

25. Safari E, Zavaran Hosseini A, Hassan Z, et al. Cytotoxic Effect of Immunotoxin Containing The Truncated Form of Pseudomonas Exotoxin A and Anti-VEGFR2 on HUVEC and MCF-7 Cell Lines. Cell J. 2014;16(2):203-210. 